\title{
Simulation of Vibration Combustion
}

\author{
Evgeny Radkevich ${ }^{1}$, and Olga Vasil'eva ${ }^{2,3^{*}}$ \\ ${ }^{1}$ Faculty of Mechanics and Mathematics, Lomonosov Moscow State University, Vorobiovy gory \\ Moscow, Russia, 119991 \\ ${ }^{2}$ Moscow State University of Civil Engineering, Yaroslavskoe shosse, 26, Moscow, 129337, Russia \\ ${ }^{3}$ D. Mendeleev University of Chemical Technology of Russia, Higher Chemical College, Miusskaya \\ Sq. 9, Moscow, Russia. 125047
}

\begin{abstract}
We consider a mathematical model of the laminar process of vibration combustion proposed recently. It allows us to simulate detonation and deflagration modes, the occurrence of which occurs depending on the structure of the standard chemical potential. For simplicity of presentation and analysis, we consider a one-dimensional mathematical model formulated for the reduced dimensionless variables for the case of a twocomponent mixture. The obtained numerical results are presented and discussed.
\end{abstract}

\section{Introduction}

We consider the mathematical model of the laminar process of vibration combustion proposed recently in the paper [1]. Currently, there is a great interest in studying these processes [1-13]. The mathematical model based on thermodynamic analysis of the combustion process. The mathematical model is a boundary value problem for a closed nonlinear system of second-order differential equations. It allows us to simulate detonation and deflagration modes. For simplicity of presentation and analysis, we consider a onedimensional mathematical model formulated for the reduced dimensionless variables for the case of a two-component mixture. The obtained numerical results are presented and discussed, which allow us to conclude that one of the two modes occurs.

\section{Problem formulation}

We consider the closed mathematical model of the laminar vibration combustion process of a two-component mixture formulated in the reduced dimensionless variables [1]

$$
\frac{\partial \rho}{\partial t}+\frac{\partial(V \rho)}{\partial x}=\varepsilon \frac{\partial^{2} \rho}{\partial x^{2}}
$$

\footnotetext{
* Corresponding author: vasiljeva.ovas@yandex.ru
} 


$$
\begin{aligned}
& \frac{\partial\left(c_{0} n v_{1} \rho\right)}{\partial t}+\frac{\partial\left(c_{0} n v_{1}^{2} \rho\right)}{\partial x}+\frac{\partial P}{\partial x}=\varepsilon \frac{\partial^{2} v_{1}}{\partial x^{2}} \\
& \frac{\partial\left(\left(1-c_{0} n\right) v_{2} \rho\right)}{\partial t}+\frac{\partial\left(\left(1-c_{0} n\right) v_{2}^{2} \rho\right)}{\partial x}+\frac{\partial P}{\partial x}=\varepsilon \frac{\partial^{2} v_{2}}{\partial x^{2}} \\
& \rho \frac{d E}{d t}+P \frac{\partial V}{\partial x}=\varepsilon \frac{\partial^{2} E}{\partial x^{2}} \\
& \rho \frac{d T}{d t}=\frac{\partial}{\partial x}\left(\lambda \frac{\partial T}{\partial x}\right)+T S W(n, T) \\
& \rho \frac{d n}{d t}=\frac{\partial}{\partial x}\left(\rho D \frac{\partial n}{\partial x}\right)-W(n, T) \\
& P=(\gamma-1) \rho E+\frac{P_{0}}{\rho_{0}} \rho+\left(-T \frac{d \mu_{0}(T)}{d T}+\mu_{0}(T)+R T\right) n \rho \\
& S=-\left(\frac{d \mu_{0}(T)}{d T}+R \ln \left(\frac{n T \rho^{2}}{n_{0} T_{0} \rho_{0}^{2}}\right)\right) n \\
& W=k_{0} \rho n e^{-\frac{10}{R T}}
\end{aligned}
$$

$V=c_{0} n v_{1}+\left(1-c_{0} n\right) v_{2}$ is the average velocity of the mixture, $v_{1}$ is the velocity of the first component, $v_{2}$ is the velocity of the second component, $P$ is the pressure, $T$ is the temperature of the mixture, $\rho$ is the density, $E$ is the internal energy and $S$ is the entropy.

We consider the problem for one-dimensional case for $x \in[0,1]$ with the following boundary conditions

$$
\begin{aligned}
& \left.\frac{\partial \rho}{\partial x}\right|_{x=0}=0,\left.\quad \frac{\partial \rho}{\partial x}\right|_{x=1}=0,\left.\quad \frac{\partial v_{1}}{\partial x}\right|_{x=0}=0,\left.\quad \frac{\partial v_{1}}{\partial x}\right|_{x=1}=0,\left.\quad \frac{\partial v_{2}}{\partial x}\right|_{x=0}=0,\left.\quad \frac{\partial v_{2}}{\partial x}\right|_{x=1}=0, \\
& \left.\frac{\partial n}{\partial x}\right|_{x=0}=0,\left.\quad \frac{\partial n}{\partial x}\right|_{x=1}=0,\left.\quad \frac{\partial E}{\partial x}\right|_{x=0}=0,\left.\quad \frac{\partial E}{\partial x}\right|_{x=1}=0, \quad T(t, 0)=2(1+0.1 t),\left.\quad \frac{\partial T}{\partial x}\right|_{x=1}=0,
\end{aligned}
$$

and initial conditions

$$
\begin{aligned}
& \rho(0, x)=1, \quad v_{1}(0, x)=0.5, \quad v_{2}(0, x)=0.5, \quad T(0, x)=2, E(0, x)=1, \\
& n(0, x)=\left\{\begin{array}{l}
0.5, \quad x \in[0,0.3) \\
0.66, x \in[0.3,0.4] \\
0.5, \quad x \in(0.4,1] .
\end{array}\right.
\end{aligned}
$$

For numerical solution problem we use the finite-difference method of second order.

\section{Numerical results}

The simulations were performed with the standard chemical potential $\mu_{0}(T)$ of various structure. At first we consider the following case of $\mu_{0}(T)$.

$\mu_{0}(T)=T_{0}\left(B-(A+R)\left(\frac{T}{T_{0}}\right)^{2}\right), \quad \frac{d \mu_{0}}{d T}=-2(R+A)\left(\frac{T}{T_{0}}\right)$.

In the figures 1-8 we can see the evolution of solution components and some process characteristics. The time cross sections are $0,0.002,0.004,0.01$ and 0.015 . 


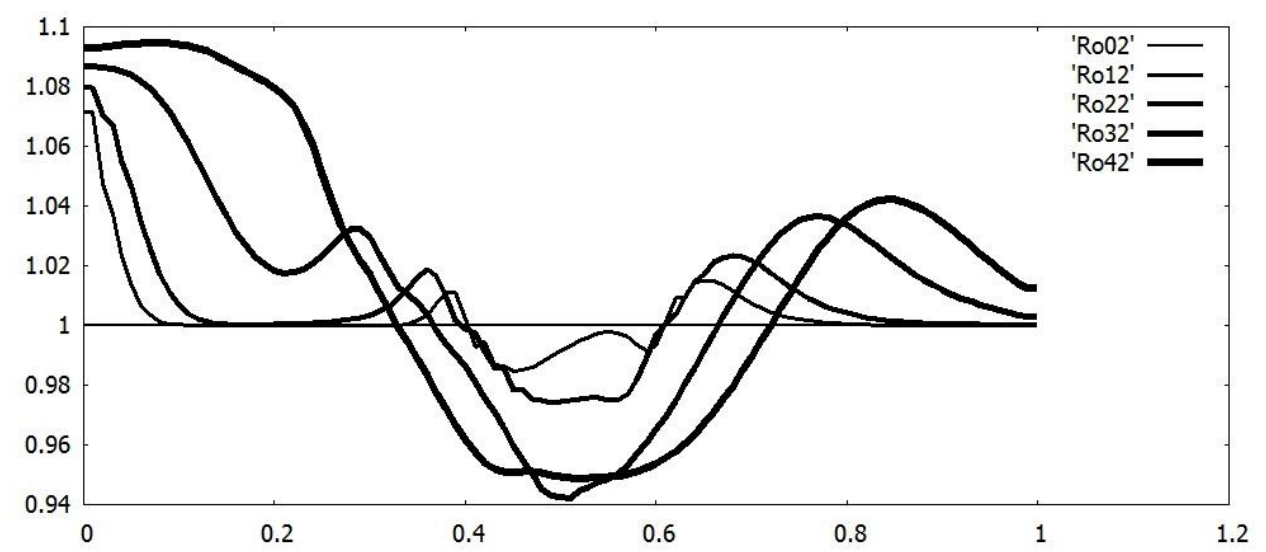

Fig. 1. The evolution of the density $\rho\left(t_{\mathrm{i}}, x\right), t_{0}=0, t_{1}=0.002, t_{2}=0.004, t_{3}=0.01, t_{4}=0.015$

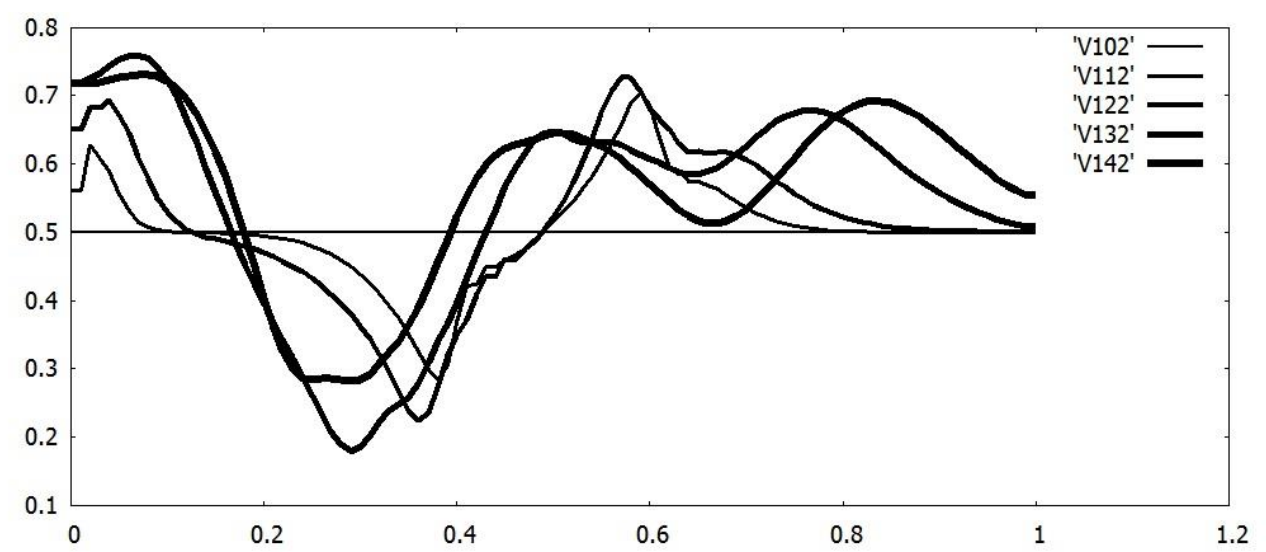

Fig.2. The evolution of the velocity $v_{1}\left(t_{\mathrm{i}}, x\right), t_{0}=0, t_{1}=0.002, t_{2}=0.004, t_{3}=0.01, t_{4}=0.015$

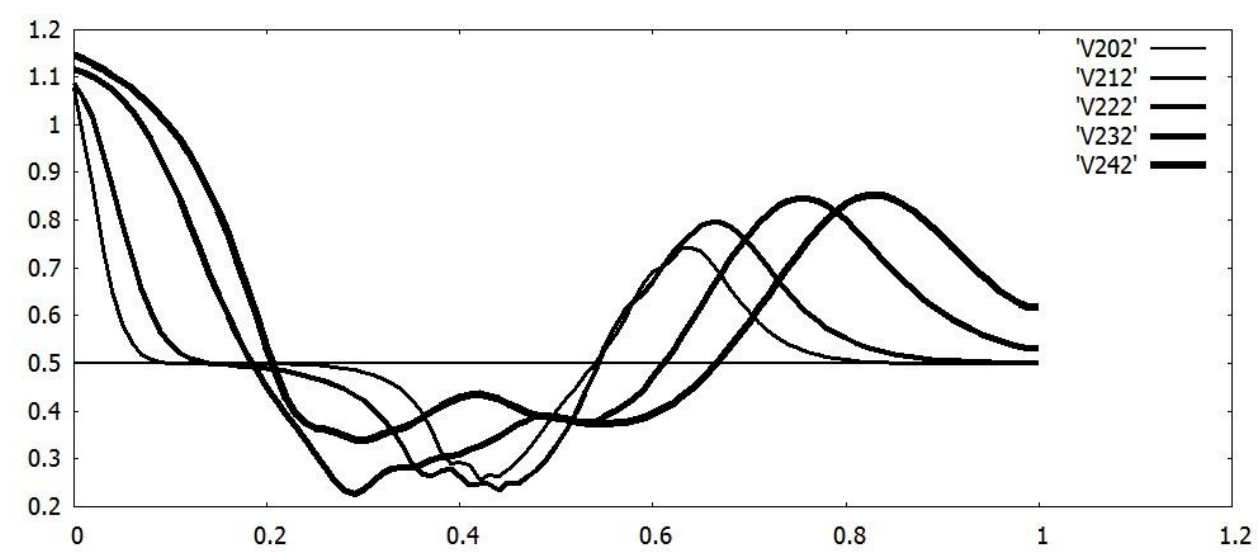

Fig. 3. The evolution of the velocity $v_{2}\left(t_{\mathrm{i}}, x\right), t_{0}=0, t_{1}=0.002, t_{2}=0.004, t_{3}=0.01, t_{4}=0.015$ 


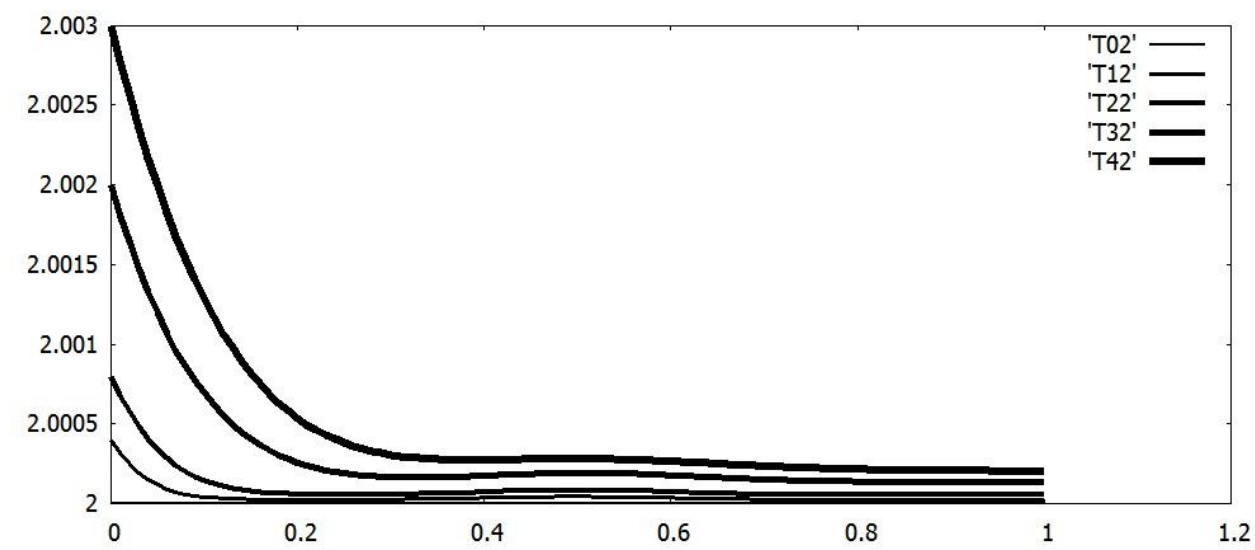

Fig. 4. The evolution of temperature $T\left(t_{\mathrm{i}}, x\right), t_{0}=0, t_{1}=0.002, t_{2}=0.004, t_{3}=0.01, t_{4}=0.015$



Fig. 5. The evolution of internal energy $E\left(t_{1}, x\right), t_{0}=0, t_{1}=0.002, t_{2}=0.004, t_{3}=0.01, t_{4}=0.015$

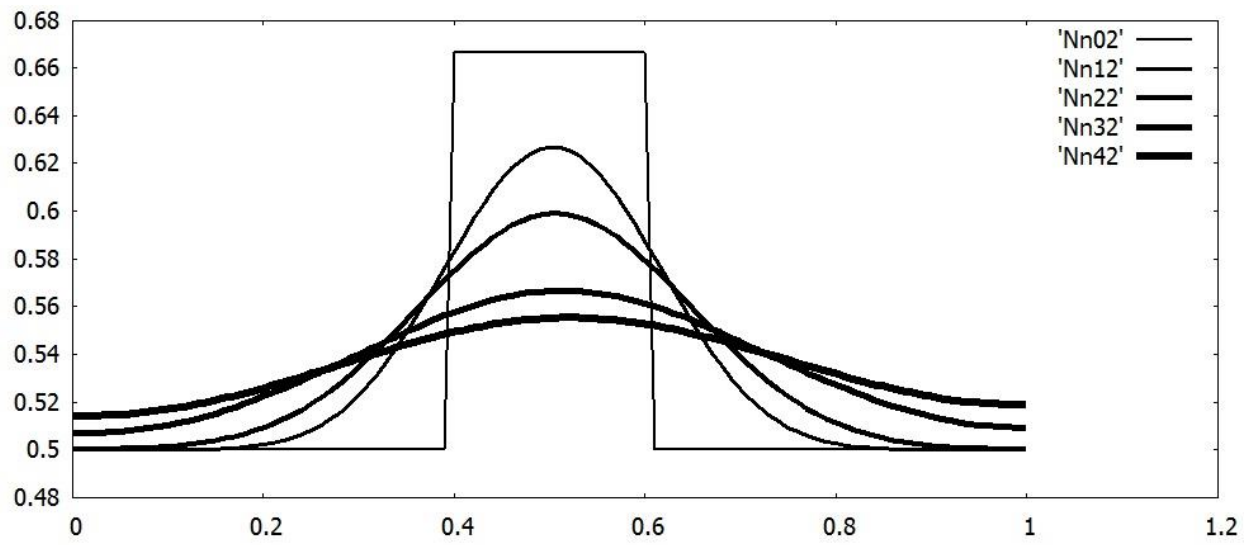

Fig. 6. The evolution of $n\left(t_{\mathrm{i}}, x\right), t_{0}=0, t_{1}=0.002, t_{2}=0.004, t_{3}=0.01, t_{4}=0.015$ 


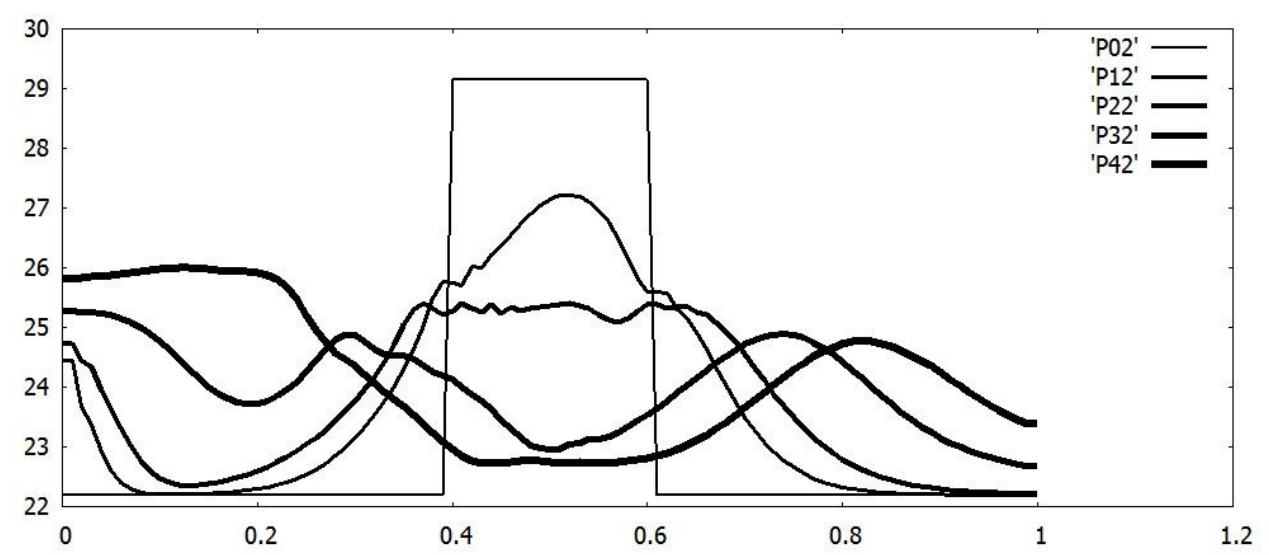

Fig. 7. The evolution of pressure $P\left(t_{1}, x\right), t_{0}=0, t_{1}=0.002, t_{2}=0.004, t_{3}=0.01, t_{4}=0.015$

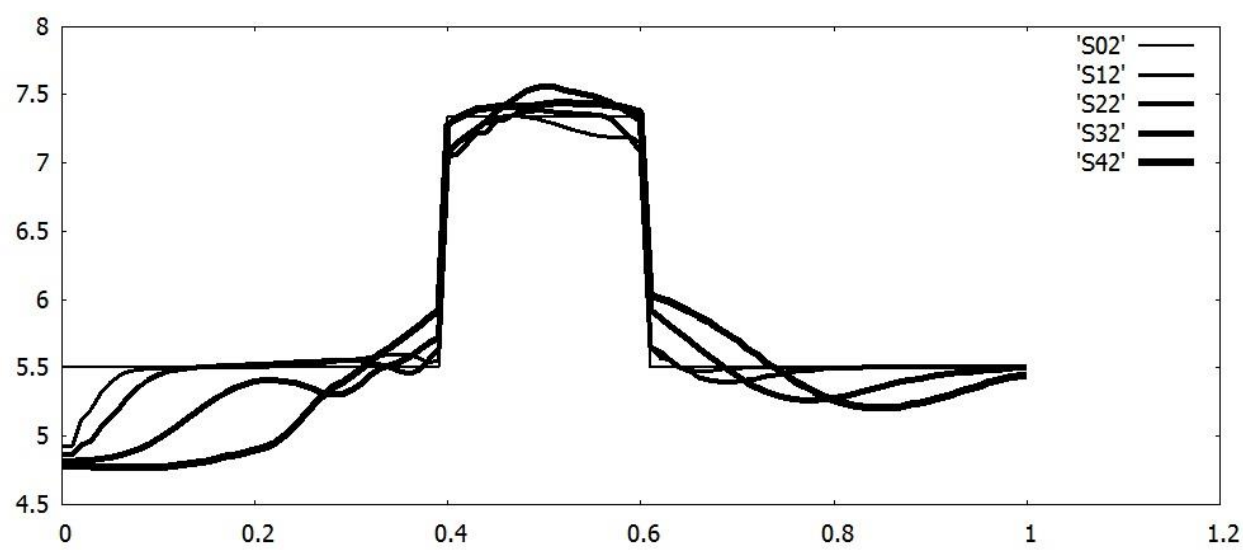

Fig. 8. The evolution of entropy $S\left(t_{i}, x\right), t_{0}=0, t_{1}=0.002, t_{2}=0.004, t_{3}=0.01, t_{4}=0.015$

In the fig. 9, 10 we can see the comparison the evolution the pressure and entropy for the first case of the standard chemical potential $\mu_{0}(T)$ and for the following standard chemical potential (the second case)

$\mu_{0}(T)=T_{0}\left(B-(A+R)\left(\frac{T}{T_{0}}\right)^{4}\right), \quad \frac{d \mu_{0}}{d T}=-4(R+A)\left(\frac{T}{T_{0}}\right)^{3}$. 


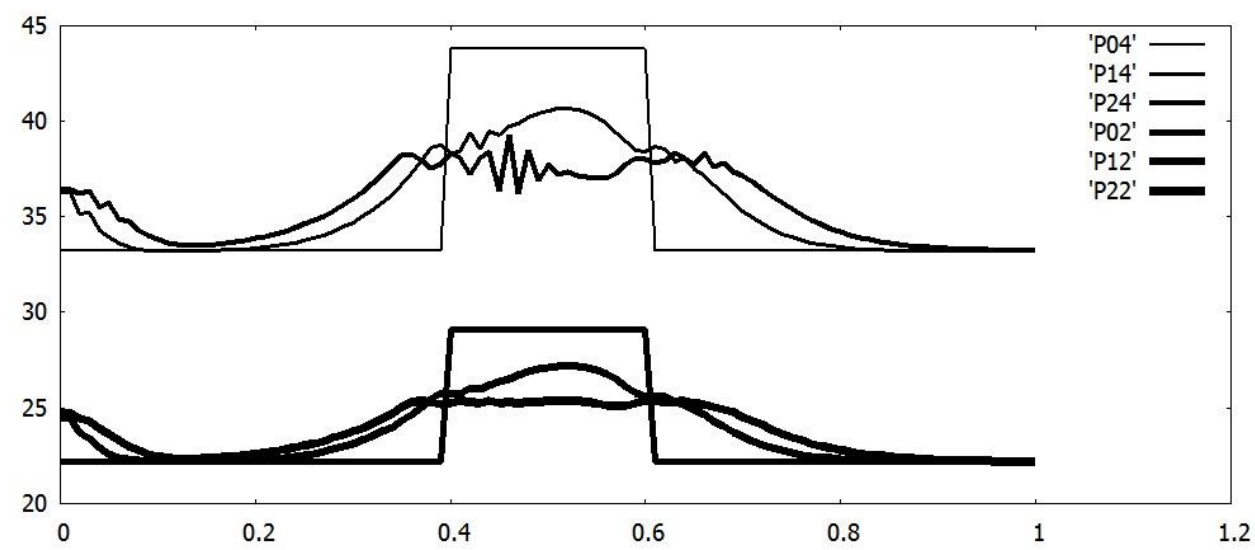

Fig. 9. The evolution of pressure $S\left(t_{\mathrm{i}}, x\right)$, for two structure of the chemical potential

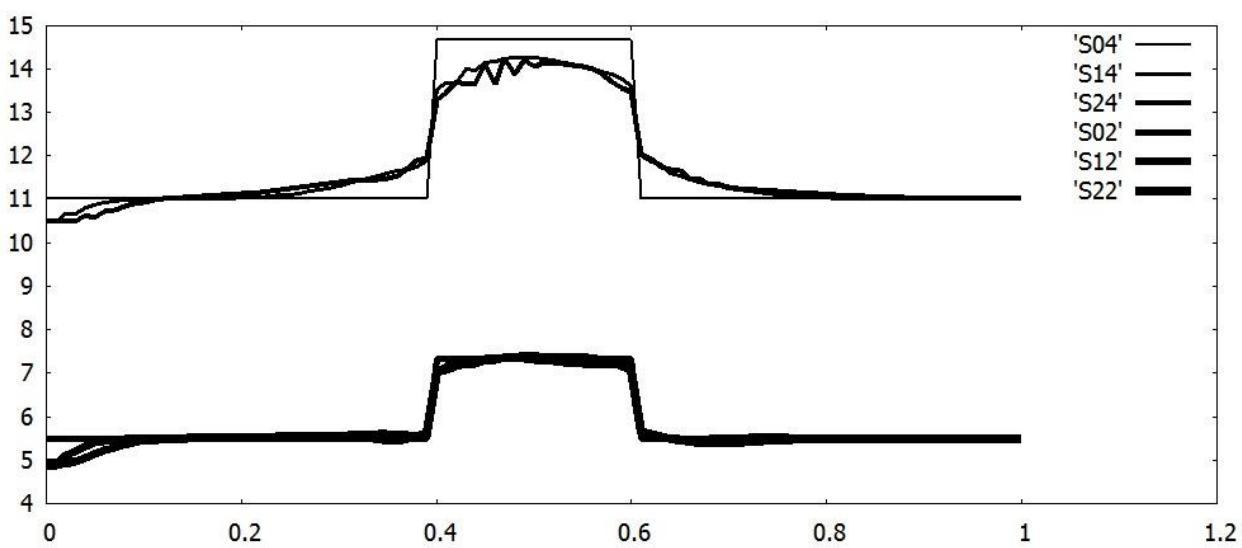

Fig. 10. The evolution of entropy $S\left(t_{i}, x\right)$, for two structure of the chemical potential

The time cross sections are $0,0.002,0.004$. Here we can see that the different combustion modes are determined by various structures of the standard chemical potential $\mu_{0}(T)$.

\section{Conclusion}

We consider the mathematical model of the laminar process of vibration combustion proposed recently. The numerical solutions were performed with the standard chemical potential $\mu_{0}(T)$ of various structure. The numerical results are presented and compared. So, the different combustion modes are determined by various structures of the standard chemical potential $\mu_{0}(T)$. The study of the process based on nonequilibrium phase transitions [14-20] is the subject of the following article.

\section{References}

1. E. Radkevich, N. Yakovlev, O. Vasil'eva, Dokl. Math, 102, 3 (2020)

2. B. Raushenbakh, Vibration Burning, Fizmatgiz, Moscow, 1961 [in Russia] 
3. E. Radkevich, N. Yakovlev, O. Vasil'eva, M.Sidorov, M.Stavrovskii, Possibility of Applying the Canh-Hilliard Theory to Mathematical Modeling of Combustion, EkoPress, Moscow, 2020 [in Russia]

4. E. Radkevich, N. Yakovlev, O. Vasil'eva, AIP Conf. Proc, 2333 (2021)

5. E. Radkevich, E. Lukashev, O. Vasil'eva, Dokl. Math, 99, 3 (2019)

6. C.Lee, R.Reitz, Int. J. Multiphase. Flow, 26 (2000)

7. S.Frolov, V.Ivanov, Deflagrative and Detonative Combustion, Torus, Moscow, 2010 [in Russia]

8. E.Shchetinkow The Physics of the Combstion Gases, Nauka, Moskow, 1965 [in Russia]

9. D.Lewis, G. Elbe, J.Chem. Phys, 2, 8 (1934)

10. Yu. Zeldovich, Kinet. Katal, 11, 3 (1961)

11. R. Reitz, Atomatization and Spray Technology, 3 (1987)

12. P. Debye, Selected Works, Nauka, Leningrad, 1987 [in Russia]

13. E. Radkevich, N. Yakovlev, O. Vasil'eva, Eurasion J. Math. and Comp. Appl, 8, 4 (2020)

14. J. Cahn, J. Hillard, J. Chem. Phys, 28, 2 (1958)

15. J. Cahn, J. Hillard, J. Chem. Phys, 30, 2 (1958)

16. J. Cahn, Acta Met, 9, 8 (1961)

17. E. Radkevich, N. Yakovlev, O. Vasil'eva, Eurasion J. Math. and Comp. Appl, 6, 2 (2018)

18. E. Radkevich, E. Lukashev, O. Vasil'eva, Eurasion J. Math. and Comp. Appl, 7, 2 (2019)

19. E. Radkevich, O. Vasil'eva, J. Math. Science, 244, 2 (2020)

20. E. Lukashev, N. Yakovlev, E. Radkevich, O. Vasil'eva, AIP Conf. Proc, 1910 (2017) 